\title{
The Activity and Safety of Anlotinib for Patients with Extremity Desmoid Fibromatosis: A Retrospective Study in a Single Institution
}

This article was published in the following Dove Press journal: Drug Design, Development and Therapy

\author{
Chuanxi Zheng* \\ Yong Zhou* \\ Yitian Wang \\ Yi Luo (D) \\ Chongqi Tu \\ Li Min (D)
}

Department of Orthopedics, West China Hospital, Sichuan University, Chengdu, Sichuan 6I004I, People's Republic of China

*These authors contributed equally to this work
Correspondence: Li Min

Department of Orthopedics, West China Hospital, Sichuan University, No. 37

Guoxuexiang, Chengdu 6I004I, People's

Republic of China

Tel +86 |37603 II 476

Fax +8602885582944

Email minliI204@scu.edu.cn
Purpose: Desmoid fibromatosis (DF) is an aggressive fibroblastic neoplasm with a high propensity for local recurrence. Although multiple therapeutic modalities seem effective for DF, the standard systemic treatment for symptomatic and progressive DF remains controversial. As targeted therapy, tyrosine kinase inhibitors have been recently reported to contribute to the treatment of DF. Thus, the purpose of this study was to assess the efficacy and safety of anlotinib, a novel multi-kinase angiogenesis inhibitor, in patients with DF.

Patients and Methods: We retrospectively collected the clinical medical records of patients with extremity DF who received anlotinib between January 2019 and January 2020 in our center. Anlotinib was started with a dose of $8 \mathrm{mg}$ daily and adjusted according to the drug-related toxicity. Tumor response was assessed by the Response Evaluation Criteria in Solid Tumors 1.1 criteria. Progression-free survival (PFS) was identified as the primary endpoint and analyzed using the Kaplan-Meier method.

Results: In total, 21 (6 male, 15 female) consecutive patients with DF were enrolled. The median medication time was nine months (Q1, Q3: 7.5, 10.5). None of the patients achieved a complete response, but eight $(38.1 \%)$ patients achieved a partial response and ten patients $(47.6 \%)$ achieved disease stability. Three (14\%) patients developed progressive disease and the 3-, 6-, and 12-month PFS rates were $95.2 \%, 90.5 \%$, and $84.0 \%$, respectively. The disease control rate was $86.0 \%(18 / 21)$ and the objective response rate was $38.1 \%(8 / 21)$. Moreover, $15 / 21(71.4 \%)$ patients achieved a reduction in tumor size, accompanied with a decrease in T2-weighted signal intensity on magnetic resonance imaging and clinical benefit.

Conclusion: Anlotinib was effective against DF with an acceptable safety profile, and significantly slowed the disease progression. Further, multicenter studies with a longer follow-up time are needed to characterize fully the clinical application of anlotinib in DF.

Keywords: desmoid fibromatosis, anlotinib, tyrosine kinase inhibitor, targeted therapy

\section{Introduction}

Desmoid fibromatosis (DF) is an intermediate fibroblastic neoplasm arising from musculoaponeurotic tissues, characterized by infiltrative growth, but without a propensity to metastasize. ${ }^{1}$ DF can be located at virtually any anatomical site; the common sites of involvement are the abdominal wall, abdominal mesentery, and extremities. $^{2}$ Among these anatomic locations, abdominal wall DFs have the most indolent course. ${ }^{3}$ In contrast, extremity DFs usually portend a higher risk of recurrence and worse outcomes, and pose more difficulties in therapeutic decisionmaking by surgeons. ${ }^{4}$ 
The National Comprehensive Cancer Network guidelines (Version 2.2020) state that asymptomatic patients can be managed appropriately by active surveillance, but for symptomatic or progressive patients, surgical management is still the primary treatment. ${ }^{2,5}$ The impact of surgical margins on local control and risk of recurrence presently remain controversial. A wide surgical excision does not yield better local tumor control, and has a high local recurrent rate of $20 \%-80 \%$, which may be associated with significant morbidity and mortality. ${ }^{6}$ Radiotherapy is recommended in patients with positive surgical margins, as well as in those with recurrent or unresectable disease. ${ }^{7,8}$ The combination of surgery and adjuvant radiotherapy has a lower local recurrent rate than surgical resection alone. ${ }^{9}$ However, clinicians must be concerned of the severe side effects of radiation, including wound complications, secondary malignancy, and growth restriction in young patients.

Systemic therapy is usually considered for symptomatic or progressive disease not amenable to surgery or radiotherapy, including antiestrogenic agents (tamoxifen, toremifene) combined with non-steroidal anti-inflammatory drugs (celecoxib, sulindac), and cytotoxic chemotherapy (doxorubicin, methotrexate and vinblastine). ${ }^{10,11}$ However, antiestrogenic treatments show low response rates, and no clear relationship with therapeutic effectiveness has been demonstrated. ${ }^{12}$ The combination of doxorubicin, methotrexate, and vinorelbine or vinblastine is associated with prolonged stable disease in patients with unresectable tumors. However, the results among different studies are variable. ${ }^{13-15}$ At the same time, continuing chemotherapy with doxorubicin may cause cumulative cardiotoxicity and potential damage to fertility in young females of childbearing ages, who comprise the dominant population of DF. ${ }^{16,17}$

As a new nonchemotherapeutic systemic treatment, tyrosine kinase inhibitors, including imatinib, sorafenib, and pazopanib, have been evaluated in patients with unresectable, progressive, or recurrent $\mathrm{DF}$, with some promising clinical results. ${ }^{18-21}$ Anlotinib is a novel tyrosine kinase inhibitor that selectively competes with vascular endothelial growth factor receptor (VEGFR)-2, -3, with a half-maximal inhibitory concentration of $0.2 \mathrm{nmol} / \mathrm{L}$ in vitro, which synchronously inhibits the activities of VEGFR-1, platelet-derived growth factor receptor (PDGFR- $\beta$ ), and hepatocyte factor receptor (c-KIT). ${ }^{22}$ Pharmacokinetic assessment has revealed that anlotinib obtains its maximum plasma concentration at $7.5 \pm 3$ hours after dosing, and then is eliminated slowly, with a half-life of $100 \pm 36$ hours. Anlotinib has exhibited encouraging antitumor effects and acceptable toxicity in advanced lung cancer and soft tissue sarcoma. ${ }^{22-25}$ This drug was approved by the Chinese Food and Drug Administration (CFDA) for the treatment of advanced non-small-cell lung cancer in 2018. However, the role of anlotinib in DF remains unknown; therefore, we retrospectively assessed the efficacy and safety of anlotinib in patients with DF treated in our center.

\section{Patients and Methods}

\section{Patient Selection and Ethical Clearance}

We retrospectively reviewed the clinical medical records of patients with DF who were treated with anlotinib between January 2019 and January 2020 in the Department of Orthopedics at West China Hospital. The inclusion criteria were as follows: (1) pathologically confirmed DF; (2) tumor located in the upper or lower extremity, including the shoulder girdle and buttock; (3) patient had progressive or symptomatic disease; and (4) patient had recurrent or primary disease that was unresectable for surgery or declined radiotherapy, chemotherapy, or surgery. Progressive disease was defined as an increase in the maximum unidimensional measurement of the lesions by more than $10 \%$ on magnetic resonance imaging (MRI) within three months. Unresectable disease was defined as follows: extensive resection of a primary or recurrent tumor was not deemed feasible due to the size or location of the lesions, or would be unacceptably morbid after extensive excision. The following patient and disease characteristics were collected: age, sex, presentation status (primary or recurrent), tumor size, tumor location, previous therapeutic history, date of initial anlotinib treatment, reason for treatment discontinuation, dose and toxicity of anlotinib, time to progression, date of death if available. All patients provided informed consent for anlotinib treatment. This study was performed according to the principles embodied in the Declaration of Helsinki and the Institutional Review Board of Sichuan University West China Hospital.

\section{Treatment and Evaluation}

All patients received anlotinib at a starting dose of $8 \mathrm{mg}$ once daily; the 2-week on/1-week off and treatment cycle lasted three weeks. Dose reduction (to $6 \mathrm{mg}$ ) was allowed if the patient showed intolerable or uncontrolled drug-related toxicity. Treatment warranted a temporary interruption if 
excessive toxicity could not be adequately controlled by dose reduction and symptomatic treatment. The dose was increased to $10 \mathrm{mg}$ once the patient had progression according to Response Evaluation Criteria in Solid Tumors (RECIST) $1.1{ }^{26}$ If a patient could not achieve stabilization or experienced uncontrolled side effects in the subsequent two-cycle treatment with $10 \mathrm{mg}$, then the patient was excluded from anlotinib treatment.

The pretreatment baseline evaluation included a physical examination, routine blood test, and imaging examination of the measurable lesions. Treatment efficacy was assessed by MRI after two treatment cycles, or more frequently in patients with substantial progression or discontinuous treatment. Follow-up visits were mainly conducted at an outpatient clinic. Tumor response was defined as a complete response (CR), partial response (PR), stable disease (SD), or progressive disease (PD) according to RECIST criteria $1.1 .^{26}$ Progression-free survival (PFS) was the endpoint and identified as the time from the start of anlotinib administration until disease progression, death, or the last follow-up. The objective response rate (ORR) was defined as the proportion of patients with $\mathrm{CR}$ and PR. Disease control rate (DCR) was defined as the proportion of patients without disease progression on record. Drug-related adverse effects were classified and graded according to the National Cancer Institute Common Terminology Criteria for Adverse Events (version 4.0).

Clinical benefit was mainly based on the assessment of pain relief and changes in physical examination findings (adjacent joint range of motion). The level of pain was subjectively reported by patients and quantitated with the use of a visual analog scale (VAS) score at the first visit. These evaluations were carried out prospectively during routine clinical follow-up, and then retrospectively identified.

Change in the tumor T2-weighted signal on MRI was objectively assessed using the modified Choi technique described by Stacchiotti et $\mathrm{al}^{27}$ that the radiologist selected the greatest cross-sectional diameter of lesions and drew the largest possible circular region of interest within the tumor. A second region of interest was drawn on the adjacent normal skeletal muscle and then the ratio between tumor and muscle was calculated. These measurements were repeated at the same location of the tumor on subsequent follow-up examinations. The cut-off date for the statistical analysis of clinical outcomes was April 30, 2020. PFS was estimated according to the Kaplan-Meier method. P-values $<0.05$ were considered significant (twosided). Statistical analysis was performed using SPSS version 22 (IBM Corporation, Armonk, NY, USA) and GraphPad Prism (version 8).

\section{Results}

\section{Patients' Characteristics}

From January 2019 and January 2020, 21 (6 male, 15 female) consecutive patients with extremity DF were enrolled. All pathologic diagnoses were confirmed by an experienced pathologist at West China Hospital. The demographics and clinical baseline characteristics are summarized in Table 1. The median age was 27 (range 16-82) years, with a female to male ratio of 2.5 . The primary anatomical location of the DF was as follows: upper $\operatorname{arm}(n=6,28.6 \%)$, gluteal region $(n=4,19.0 \%)$, scapular region $(\mathrm{n}=2,9.5 \%)$, popliteal region $(\mathrm{n}=2$,

Table I Patient Demographics and Clinical Baseline Characteristics

\begin{tabular}{|c|c|c|}
\hline Characteristics & & Patients (\%) \\
\hline \multirow[t]{2}{*}{ Age } & Median & 27 \\
\hline & Range & $16-82$ \\
\hline \multirow[t]{2}{*}{ Gender } & Female & 15 (7I.4\%) \\
\hline & Male & $6(28.6 \%)$ \\
\hline \multirow[t]{9}{*}{ Tumor location } & Upper arm & $6(28.6 \%)$ \\
\hline & Gluteal region & $4(19.0 \%)$ \\
\hline & Scapular region & $2(9.5 \%)$ \\
\hline & Popliteal region & $2(9.5 \%)$ \\
\hline & Hand & $2(9.5 \%)$ \\
\hline & Thigh & $2(9.5 \%)$ \\
\hline & Foot & I (4.8\%) \\
\hline & Forearm & I (4.8\%) \\
\hline & Axillary region & I (4.8\%) \\
\hline \multirow{2}{*}{$\begin{array}{l}\text { Resectable } \\
\text { lesions }\end{array}$} & Untreated & $3(14.3 \%)$ \\
\hline & Recurrent & $4(19.0 \%)$ \\
\hline \multirow{2}{*}{$\begin{array}{l}\text { Unresectable } \\
\text { lesions }\end{array}$} & Untreated & $5(23.8 \%)$ \\
\hline & Recurrent & $9(42.9 \%)$ \\
\hline \multirow[t]{3}{*}{ Surgery history } & None & $8(38.1 \%)$ \\
\hline & Recurrence following surgery & 7 (33.3\%) \\
\hline & $\begin{array}{l}\text { Recurrence after two or } \\
\text { more surgery }\end{array}$ & $6(28.6 \%)$ \\
\hline \multirow{2}{*}{$\begin{array}{l}\text { Radiotherapy } \\
\text { history }\end{array}$} & Yes & $3(14.3 \%)$ \\
\hline & No & $18(85.7 \%)$ \\
\hline \multirow{2}{*}{$\begin{array}{l}\text { Chemotherapy } \\
\text { history }\end{array}$} & Yes & $6(28.6 \%)$ \\
\hline & No & 15 (7I.4\%) \\
\hline
\end{tabular}


$9.5 \%)$, hand $(n=2,9.5 \%)$, thigh $(n=2,9.5 \%)$, foot $(n=1$, $4.8 \%)$, axillary region $(n=1,4.8 \%)$, and forearm $(\mathrm{n}=1,4.8 \%)$.

Our patient cohort had been heavily pretreated, with the exception of eight patients (38.1\%) who did not receive any systemic or surgical therapy before treatment. Seven patients $(33.3 \%)$ were locally recurrent following surgery, and six patients $(28.6 \%)$ were re-recurrent following two or more surgical procedures, with a median number of prior surgeries of 3 (range, 2-7). Five untreated patients (23.8\%) and nine recurrent patients $(42.9 \%)$ were identified as having unresectable diseases involving the vital neurovascular structures or without acceptable morbidity after resection. Three untreated patients $(14.3 \%)$ and four recurrent patients $(19 \%)$ were identified as having resectable diseases, but refused surgery. Additionally, nine patients had received other nonsurgical treatments, including radiotherapy in three patients $(14.3 \%)$ and chemotherapy in six patients $(28.6 \%)$. The most commonly used chemotherapy agents were doxorubicin, methotrexate, and vinblastine.

\section{Treatment Outcomes}

The clinical features of anlotinib therapy in this study are detailed in Table 2. All patients were followed up for median time of 11 months (Q1, Q3: 9.5, 12.5), and no patient died of the disease. In all 21 patients, the initial anlotinib dose of $8 \mathrm{mg}$ daily was administered, but two patients $(9.5 \%)$ had to reduce the dose due to intolerable drug-related toxicity. None of the patients achieved CR. However, eight (38.1\%) patients had PR. Ten patients had SD at least lasting more than six months, yielding an overall DCR of $86 \%(18 / 21)$ and ORR of $38.1 \%(8 / 21)$ for DF. Three patients (14\%) developed PD and the 3-, 6-, and 12-month PFS rates were 95.2\%, 90.5\%, and $84.0 \%$, respectively. However, the median PFS had not yet been reached by the time of analysis. Of these three patients, two patients with re-recurrent DF following two more surgeries had progression after anlotinib therapy with a dose of $8 \mathrm{mg}$ and received two cycles of additional treatment. When this treatment did not show a potential to stabilize tumor growth, they subsequently underwent amputation surgery due to treatment failure. One patient with untreated DF had disease progression after eight months of anlotinib therapy, but she refused to receive additional treatment with a high dose or alternative therapy, and ultimately chose amputation surgery.

The tumor size in 15 patients $(71.4 \%)$ was reduced after anlotinib therapy. The median tumor shrinkage in all

Table 2 Clinical Characteristics of Patient with DF Treated with Anlotinib

\begin{tabular}{|c|c|c|c|c|c|c|c|c|c|c|}
\hline Patients & Age & Gender & $\begin{array}{l}\text { Tumor } \\
\text { Location }\end{array}$ & $\begin{array}{l}\text { Number of } \\
\text { Surgery }\end{array}$ & $\begin{array}{l}\text { Initial } \\
\text { Dose }\end{array}$ & $\begin{array}{l}\text { Medication } \\
\text { Time (M) }\end{array}$ & $\begin{array}{l}\text { Tumor Size } \\
\text { Change }\end{array}$ & $\begin{array}{l}\text { T2-Weighted } \\
\text { MRI } \\
\text { Change }\end{array}$ & $\begin{array}{l}\text { RECIST } \\
\text { I.I } \\
\text { Response }\end{array}$ & $\begin{array}{l}\text { PFS } \\
\text { (M) }\end{array}$ \\
\hline I & 21 & Female & Upper arm & I & $8 \mathrm{mg}$ & 13 & $-64 \%$ & $-58 \%$ & PR & NA \\
\hline 2 & 29 & Female & Gluteal region & 3 & $8 \mathrm{mg}$ & 14 & $8 \%$ & $-10 \%$ & SD & NA \\
\hline 3 & 82 & Female & Thigh & 0 & $8 \mathrm{mg}$ & 8 & $37 \%$ & $5 \%$ & PD & 8 \\
\hline 4 & 26 & Female & Popliteal region & 2 & $8 \mathrm{mg}$ & 9 & $-23 \%$ & $-37 \%$ & SD & NA \\
\hline 5 & 25 & Female & Hand & I & $8 \mathrm{mg}$ & 10 & $-45 \%$ & $-62 \%$ & PR & NA \\
\hline 6 & 30 & Female & Forearm & 0 & $8 \mathrm{mg}$ & 9 & $-28 \%$ & $-32 \%$ & SD & NA \\
\hline 7 & 23 & Female & Gluteal region & I & $8 \mathrm{mg}$ & 9 & $-50 \%$ & $-70 \%$ & PR & NA \\
\hline 8 & 40 & Female & Gluteal region & I & $8 \mathrm{mg}$ & 13 & $-14 \%$ & $-64 \%$ & SD & NA \\
\hline 9 & 36 & Female & Upper arm & 0 & $8 \mathrm{mg}$ & 11 & $-56 \%$ & $-55 \%$ & PR & NA \\
\hline 10 & 21 & Female & Hand & 0 & $8 \mathrm{mg}$ & 9 & $-20 \%$ & $-27 \%$ & SD & NA \\
\hline 11 & 72 & Female & Upper arm & 0 & $8 \mathrm{mg}$ & 9 & $-19 \%$ & $-56 \%$ & SD & NA \\
\hline 12 & 21 & Female & Popliteal region & I & $8 \mathrm{mg}$ & 8 & $-22 \%$ & $-60 \%$ & SD & NA \\
\hline 13 & 27 & Female & Upper arm & 0 & $8 \mathrm{mg}$ & 8 & $-78 \%$ & $-84 \%$ & PR & NA \\
\hline 14 & 28 & Female & Upper arm & I & $8 \mathrm{mg}$ & 9 & $-61 \%$ & $-70 \%$ & PR & NA \\
\hline 15 & 55 & male & Thigh & 3 & $8 \mathrm{mg}$ & 7 & $29 \%$ & $10 \%$ & PD & 5 \\
\hline 16 & 24 & male & Gluteal region & 7 & $8 \mathrm{mg}$ & 8 & $6 \%$ & $-13 \%$ & SD & NA \\
\hline 17 & 27 & male & Scapular region & I & $8 \mathrm{mg}$ & 8 & $-78 \%$ & $-62 \%$ & PR & NA \\
\hline 18 & 16 & male & Foot & 4 & $8 \mathrm{mg}$ & 6 & $35 \%$ & $5 \%$ & PD & 4 \\
\hline 19 & 40 & male & Scapular region & 0 & $8 \mathrm{mg}$ & 6 & $5 \%$ & $-8 \%$ & SD & NA \\
\hline 20 & 23 & Female & Upper arm & 2 & $8 \mathrm{mg}$ & 6 & $-40 \%$ & $-80 \%$ & PR & NA \\
\hline 21 & 38 & Female & Axillary region & 0 & $8 \mathrm{mg}$ & 7 & $-25 \%$ & $-64 \%$ & SD & NA \\
\hline
\end{tabular}

Abbreviations: PR, partial response; SD, stable disease; PD, progressive disease; PFS, progression-free survival; NA, not achieved. 


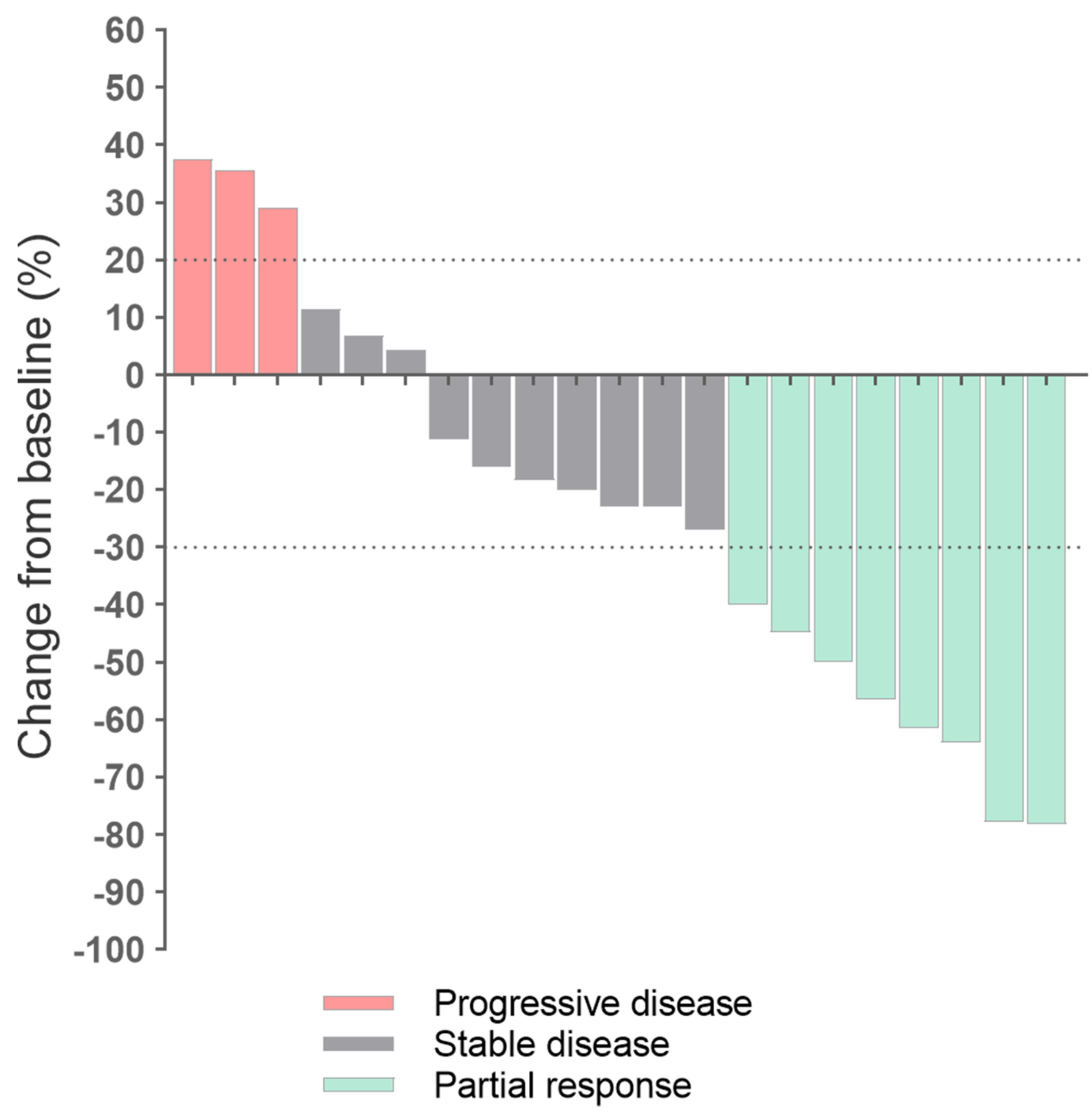

Figure I The tumor changes from baseline in patients with DF treated with anlotinib.

PR patients was 59.0\% (Q1, Q3: 46.2\%, 74.5\%), and seven SD patients obtained tumor reduction, with a median tumor shrinkage of $20.0 \%$ (Q1, Q3: 16.0\%, $23.0 \%$ ) (Figure 1). A 27-year-old patient with progressive DF and eight months of treatment experience presented with a typical therapeutic course, which is shown in Figure 2. Intriguingly, a signal decrease in the lesions on T2-weighted MRI was observed in all patients with tumor reduction. A significant decrease in the signal value was observed in all patients with $P R$, and the mean relative decrease was $67.6 \%$. Seven patients with $\mathrm{SD}$ showed a reduction in the signal value, with a mean relative decrease of $44.4 \%$. There was no statistical difference between patients with $\operatorname{PR}$ and $\operatorname{SD}(p=0.075)$. Three patients with PD showed an increase in the signal value, with a median relative increase of $7 \%$. When dichotomized to patients with $\mathrm{PR} / \mathrm{SD}$ vs patients with PD, these differences were statistically significant $(p<0.05)$. At the same time, all patients with tumor reduction described a clinical benefit from treatment in terms of pain palliation and improved mobility of the adjacent joint. All patients with PR or SD subjectively reported pain relief after treatment. The mean pretreated VAS score was 5 and the mean postoperative VAS score was 2. A significant improvement in the VAS score was observed after anlotinib treatment. Additionally, elbow mobility was improved in six patients with PR/SD, metacarpophalangeal joint mobility was improved in two patients with $\mathrm{PR} / \mathrm{SD}$, hip mobility was improved in two patients with $\mathrm{PR} / \mathrm{SD}$, knee mobility was improved in two patients with SD, shoulder mobility was improved in two patients with $\mathrm{PR} / \mathrm{SD}$, and wrist mobility was improved in one patient with SD (Table 3). Due to the diversity of the joints with mobility improvement, we did not perform a further statistical analysis of the improvement in a single joint. 

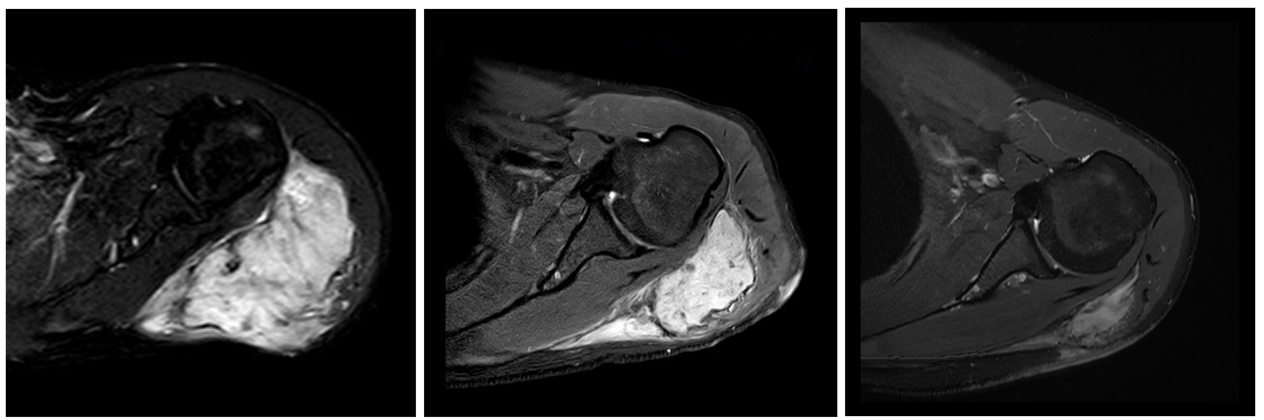

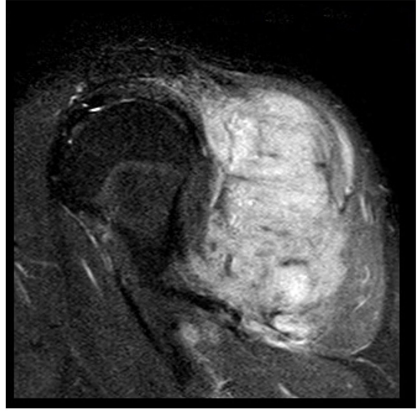

Baseline

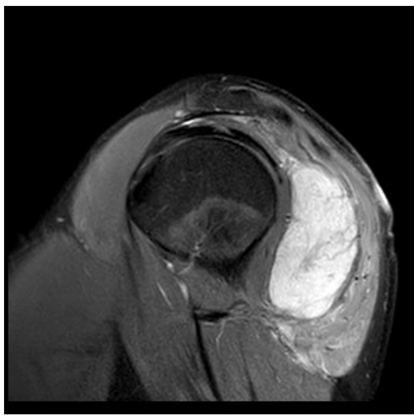

4 months

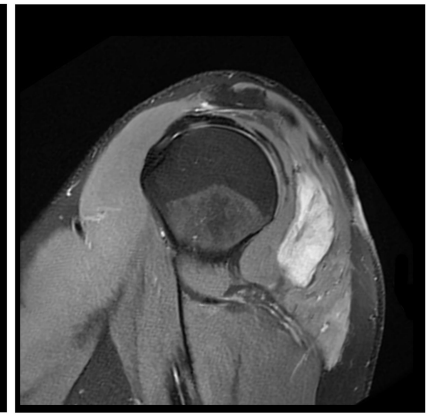

8 months

Figure 2 A 27-year-old patient with the partial disease after eight months of anlotinib treatment.

\section{Safety and Toxicity}

The median duration of medication was nine months (Q1, Q3: $7.5,10)$. The drug-related toxicities encountered during the study are shown in Table 4. The majority of adverse events comprised hand-foot skin syndrome $(n=8,38.1 \%)$, skin hypopigmentation $(\mathrm{n}=8,38.1 \%)$, paramenia $(\mathrm{n}=7,33.3 \%)$, nausea $(\mathrm{n}=5,23.8 \%)$, and diarrhea $(\mathrm{n}=5,23.8 \%)$. These adverse events were generally mild (grades 1-2) and well controlled with the support of symptomatic treatment. The grade-3 adverse events comprised hand-foot skin syndrome $(\mathrm{n}=1$, $4.7 \%)$ and vomiting $(\mathrm{n}=1,4.7 \%)$, which were remitted by dose adjustments. No grade- 4 adverse events or drug-related death occurred. Of note, paramenia occurred in seven patients, accounting for $47 \%$ of the female patients, which was considered as a tolerable side effect by the patients.

\section{Discussion}

The present retrospective study was the first to describe the efficacy and safety of anlotinib in the management of extremity DFs. At the same time, we also provided evidence suggesting that DF could obtain favorable disease control under antiangiogenic therapy.

Anti-angiogenesis, as one of the systemic therapies, has been regarded an important part of DF treatment, particularly for cases with unresectable, progressive, or recurrent lesions. Imatinib was the first agent employed against aggressive DF, which has shown a high rate of stabilization $(60-80 \%)$ despite rather low response rates (6-19\%), with a mild to moderate toxicity profile. ${ }^{28-30} \mathrm{In}$ a retrospective study involving eight patients, three patients obtained PR and durable disease stabilization was obtained in five patients after pazopanib administration, with a median PFS of 13.5 (range, 5-36) months. ${ }^{19}$ A retrospective, non-randomized study revealed that the use of sorafenib resulted in PR in $25 \%$ of patients and disease stabilization in $70 \%$ of patients. ${ }^{20}$ Recently, in a randomized, double-blind, phase $ш$ trial, sorafenib resulted in an ORR of $33 \%$, and the best response was CR; of note, $20 \%$ of the patients with placebo had spontaneous disease regression, rendering it challenging to evaluate the actual validity of tyrosine kinase inhibitor use in the treatment of DF. ${ }^{21}$ However, a larger proportion of the patients in the placebo group than in sorafenib group suffered disease progression (63\% vs 12\%). Additionally, the one-year and two-year PFS in patients with sorafenib administration was $89 \%$ and $81 \%$, respectively, which was substantially better than that in the placebo group. ${ }^{21}$ In the present study, the one-year PFS rate in patients with extremity DF was $84.0 \%$, which is similar to that for sorafenib. $^{21}$ We speculate that the improvement in PFS attributed to both sorafenib and anlotinib is related to 
Table 3 Changes of Joint Mobility and Pain Level of Patient with DF After Anlotinib Treatment

\begin{tabular}{|c|c|c|c|c|c|c|c|c|c|c|}
\hline \multirow[t]{2}{*}{ Patients } & \multirow[t]{2}{*}{ Tumor Site } & \multirow{2}{*}{$\begin{array}{l}\text { Involved } \\
\text { Joint }\end{array}$} & \multirow{2}{*}{$\begin{array}{l}\text { RECIST } \\
\text { I.I } \\
\text { Response }\end{array}$} & \multirow{2}{*}{$\begin{array}{l}\text { Pre/Post- } \\
\text { Treated } \\
\text { Pain Level } \\
\text { (VAS) }\end{array}$} & \multicolumn{6}{|c|}{ Pre/Post-Treated Range of Motion $\left({ }^{\circ}\right)$} \\
\hline & & & & & Flexion & Extension & Abduction & Adduction & $\begin{array}{l}\text { External } \\
\text { Rotation }\end{array}$ & $\begin{array}{l}\text { Internal } \\
\text { Rotation }\end{array}$ \\
\hline 1 & Upper arm & Elbow & PR & $4 / 2$ & $90 / 120$ & $0 / 0$ & NA & NA & NA & NA \\
\hline 2 & Gluteal region & Hip & SD & $5 / 3$ & $60 / 60$ & $5 / 0$ & $30 / 20$ & $20 / 20$ & $30 / 30$ & $40 / 40$ \\
\hline 3 & Thigh & Hip & PD & $5 / 4$ & I5/NA & O/NA & 20/NA & $5 / \mathrm{NA}$ & 40/NA & 40/NA \\
\hline 4 & Popliteal region & Knee & SD & $5 / 3$ & $50 / 90$ & $20 / 15$ & NA & NA & $30 / 30$ & $10 / 10$ \\
\hline 5 & Hand & MCP joint & PR & $6 / 1$ & $30 / 70$ & $5 / 30$ & NA & NA & NA & NA \\
\hline 6 & Forearm & Wrist & SD & $6 / 2$ & $20 / 50$ & $10 / 30$ & NA & NA & $25 / 50^{a}$ & $40 / 60^{b}$ \\
\hline 7 & Gluteal region & Hip & PR & $5 / 3$ & $60 / 90$ & $0 / 10$ & $15 / 20$ & $10 / 20$ & $30 / 40$ & $30 / 40$ \\
\hline 8 & Gluteal region & Hip & SD & $6 / 1$ & $90 / 130$ & $10 / 20$ & $10 / 30$ & $20 / 20$ & $40 / 40$ & $40 / 40$ \\
\hline 9 & Upper arm & Elbow & PR & $7 / 1$ & $60 / 110$ & $10 / 0$ & NA & NA & NA & NA \\
\hline 10 & Hand & MCP joint & SD & $4 / 3$ & $40 / 60$ & $10 / 20$ & NA & NA & NA & NA \\
\hline 11 & Upper arm & Elbow & SD & $3 / 0$ & $80 / 120$ & $20 / 5$ & NA & NA & NA & NA \\
\hline 12 & Popliteal region & Knee & SD & $6 / 2$ & $90 / 130$ & $10 / 0$ & NA & NA & $30 / 30$ & $10 / 10$ \\
\hline 13 & Upper arm & Elbow & PR & $3 / 0$ & $110 / 130$ & $10 / 5$ & NA & NA & NA & NA \\
\hline 14 & Upper arm & Elbow & PR & $7 / 1$ & $60 / 130$ & $5 / 0$ & NA & NA & NA & NA \\
\hline 15 & Thigh & Knee & PD & $4 / 3$ & $60 / \mathrm{NA}$ & IO/NA & NA & NA & $30 / \mathrm{NA}$ & I0/NA \\
\hline 16 & Gluteal region & Hip & SD & $3 / 1$ & $100 / 100$ & $10 / 10$ & $10 / 10$ & $20 / 20$ & $40 / 40$ & $40 / 40$ \\
\hline 17 & Scapular region & Shoulder & PR & $6 / 2$ & $50 / 90$ & $20 / 40$ & $30 / 60$ & $10 / 40$ & $40 / 60$ & $50 / 70$ \\
\hline 18 & Foot & MTP joint & PD & $5 / 6$ & 20/NA & I5/NA & NA & NA & NA & NA \\
\hline 19 & Scapular region & Shoulder & SD & $6 / 3$ & $70 / 70$ & $10 / 10$ & $20 / 20$ & $20 / 20$ & $30 / 30$ & $70 / 70$ \\
\hline 20 & Upper arm & Elbow & PR & $5 / 1$ & $90 / 110$ & $30 / 10$ & NA & NA & NA & NA \\
\hline 21 & Axillary region & Shoulder & SD & $4 / 2$ & $90 / 90$ & $30 / 40$ & $40 / 50$ & $30 / 30$ & $10 / 20$ & $30 / 40$ \\
\hline
\end{tabular}

Notes: aMeans range of pronation. ${ }^{b}$ Means range of supination.

Abbreviations: NA, not achieved, MCP, metacarpophalangeal; MTP, metatarsophalangeal.

multi-target nature of these tyrosine kinase inhibitors for VEGFR, PDGFR- $\beta$, c-KIT, leading to the inhibition of tumor angiogenesis and tumor cell proliferation. ${ }^{31,32}$

Given that DF is a locally infiltrative neoplasm without metastatic potential, the main aim of treatment is to relieve symptoms and improve the quality of life. To balance the efficacy of drug use against long-term drug-related side effects, we chose a treatment protocol with a starting dose of anlotinib ( $8 \mathrm{mg}$ daily) that was lower than the dose used in other types of soft tissue sarcomas. ${ }^{23}$ Regarding safety, the drug-related toxicity of anlotinib observed in the present study was consistent with that described in previous studies of anlotinib in patients with refractory metastatic soft-tissue sarcoma. ${ }^{23,33}$ Most adverse events were mild to moderate, and were well controlled by palliative treatment. There were no grade- 4 adverse events in the present study, although two patients $(9.5 \%)$ suffered grade- 3 adverse events, comprising hand-foot skin syndrome and vomiting. Ultimately, these two patients received a dose reduction, without discontinuing anlotinib treatment, and none of the patients dropped out because of uncontrolled adverse events. In contrast, $20 \%$ to $45 \%$ of patients discontinued the treatment of sorafenib or imatinib because of drug-related toxicities. ${ }^{29,34}$ Furthermore, $12.5 \%$ of patients interrupted pazopanib for uncontrollable grade-3 hypertension. ${ }^{19}$ In a previous report, the grade- 4 events associated with sorafenib included thrombocytopenia and anemia, and one patient died from disease-related bowel perforation. ${ }^{21}$ The overall toxicity of anlotinib was relatively well tolerated in patients with $\mathrm{DF}$, in comparison to sorafenib, imatinib, pazopanib.

Currently, an accurate evaluation of the treatment response to systematic therapy for DF is in evolution. Due to its unique histological components, the collagenization of DF usually indicates maturation and biological quiescence, presenting a low $\mathrm{T} 2$-weighted signal on MRI. ${ }^{35}$ Teixeira et al reported that a lower T2-weighted signal intensity in lesions than in the adjacent muscle showed a general tendency for stability or regression, whereas a progressive tendency was observed in patients with a T2-weighted signal intensity higher in lesions than in muscle. ${ }^{36}$ Sheth et al reported that a decrease in $\mathrm{T} 2$ hyperintensity was observed after systematic therapy (including cytotoxic chemotherapy, targeted therapy) in about $54 \%$ of patients with $\mathrm{PR} / \mathrm{SD}$, but not in patients 
Table 4 Adverse Events of Patients with DF During Anlotinib Treatment

\begin{tabular}{|l|l|l|l|l|}
\hline Adverse Event & $\begin{array}{l}\text { Total, } \\
\text { n (\%) }\end{array}$ & Grade I & Grade2 & Grade3-4 \\
\hline Hand foot skin & $8(38.1 \%)$ & 2 & 5 & 1 \\
syndrome & & & & \\
Skin hypopigmentation & $8(38.1 \%)$ & 6 & 2 & 0 \\
Paramenia & $7(33.3 \%)$ & 5 & 2 & 0 \\
Nausea & $5(23.8 \%)$ & 3 & 2 & 0 \\
Diarrhea & $5(23.8 \%)$ & 3 & 2 & 0 \\
Local pain & $4(19.0 \%)$ & 3 & 1 & 0 \\
Oral ulcers & $4(19.0 \%)$ & 4 & 0 & 0 \\
Epistaxis & $4(19.0 \%)$ & 3 & 1 & 0 \\
Hair hypopigmentation & $4(19.0 \%)$ & 4 & 0 & 0 \\
Insomnia & $3(14.1 \%)$ & 2 & 1 & 0 \\
Hypertension & $3(14.3 \%)$ & 3 & 0 & 0 \\
Vomiting & $2(9.5 \%)$ & 0 & 1 & 1 \\
Rash & $2(9.5 \%)$ & 2 & 0 & 0 \\
Pharyngalgia & $2(9.5 \%)$ & 2 & 0 & 0 \\
Gingival bleeding & $2(9.5 \%)$ & 2 & 0 & 0 \\
Fatigue & $2(9.5 \%)$ & 1 & 1 & 0 \\
Anorexia & $1(4.7 \%)$ & 1 & 0 & 0 \\
\hline
\end{tabular}

with PD. ${ }^{37}$ Seven patients in the present study had minor tumor reduction (11.2-29.0\%), but showed a decrease in hyperintensity on T2-weighted MRI, indicating a portion of patients may benefit from anlotinib treatment. Moreover, we observed that the joint range of motion in seven patients with SD had been improved, even though no striking decreases were seen in the tumor size. These patients also synchronously described a subjective diminishment in pain lasting months after anlotinib treatment. Therefore, merely measuring the tumor size seems insufficient to evaluate the treatment response in DF, and the size-based RECIST 1.1 criteria may not be adequately sensitive to assess treatment efficacy in DF. Data from the present study suggest that MRI imaging changes and physical examination findings should be incorporated in further assessments of the treatment response to targeted therapy.

Additionally, we acknowledge several limitations of the present study. As a retrospective study, not all possible clinical data could be reliably retrieved from the records, which might be prone to recall bias. Meanwhile, the medication duration in the present study was not long enough to observe some subsequent results, and the appropriate duration of anlotinib treatment remains unknown. The present study did not enroll patients with abdominal wall, intraabdominal, or trunk lesions; further studies with more comprehensive samples are necessary to determine fully the clinical efficacy and safety of anlotinib in patients with DF. Finally, the mechanism of action of anlotinib in DF is not known, and the present study was limited to clinical evaluations, without a relevant molecular mechanism investigation, such as catenin beta-1 (CTNNB1) or the adenomatous polyposis coli (APC) mutation status, reflecting activation of $\mathrm{Wnt} / \beta$ catenin pathway.

\section{Conclusion}

In summary, the present study provides the first evidence of the activity and safety of anlotinib in patients with extremity DF. Although the evidence level of the present study may be preliminary, patients treated with anlotinib indeed had a significant improvement in PFS. Additionally, anlotinibinduced toxicities were well tolerated for most patients with DF. Certainly, further long-term randomized controlled trials with larger sample sizes are needed to characterize fully the clinical application of anlotinib in DF.

\section{Ethical Statement}

To preserve patient confidentiality and privacy, patient data have been de-identified before analysis. This retrospective study was performed using data from anonymized patients who received anlotinib treatment between January 2019 and January 2020. Because of the nature of retrospective design and patient anonymization, the ethical board of Sichuan University West China Hospital approved the retrospective study.

\section{Funding}

This work was supported, in part, by the National Natural Science Foundation of China (Nos. 81702664 and 81801852) and the National Key Research and Development Program of China (Nos. 2016YFC1102003 and 2017YFB0702604).

\section{Disclosure}

The authors report no conflicts of interest in this work.

\section{References}

1. Organization WH, Cancer IAfRo. WHO Classification of Tumours of Soft Tissue and Bone. 2013.

2. Ganeshan D, Amini B, Nikolaidis P, Assing M, Vikram R. Current update on desmoid fibromatosis. J Comput Assist Tomogr. 2019;43 (1):29-38. doi:10.1097/RCT.0000000000000790

3. Bonvalot S, Ternes N, Fiore M, et al. Spontaneous regression of primary abdominal wall desmoid tumors: more common than previously thought. Ann Surg Oncol. 2013;20(13):4096-4102. doi:10.1245/s10434-013-3197-x 
4. Gronchi A, Casali PG, Mariani L, et al. Quality of surgery and outcome in extra-abdominal aggressive fibromatosis: a series of patients surgically treated at a single institution. J Clin Oncol. 2003;21(7):1390-1397. doi:10.1200/JCO.2003.05.150

5. Briand S, Barbier O, Biau D, et al. Wait-and-see policy as a first-line management for extra-abdominal desmoid tumors. J Bone Joint Surg Am. 2014;96(8):631-638. doi:10.2106/JBJS.M.00988

6. Wang YF, Guo W, Sun KK, et al. Postoperative recurrence of desmoid tumors: clinical and pathological perspectives. World J Surg Oncol. 2015;13(1):26. doi:10.1186/s12957-015-0450-8

7. Gluck I, Griffith KA, Biermann JS, Feng FY, Lucas DR, Ben-Josef E. Role of radiotherapy in the management of desmoid tumors Int J Radiat Oncol Biol Phys. 2011;80(3):787-792. doi:10.1016/j. ijrobp.2010.02.053

8. Kasper B, Baumgarten C, Garcia J, et al. An update on the management of sporadic desmoid-type fibromatosis: a European consensus initiative between sarcoma patients EuroNet (SPAEN) and European organization for research and treatment of cancer (EORTC)/soft tissue and bone sarcoma group (STBSG). Ann Oncol. 2017;28 (10):2399-2408. doi:10.1093/annonc/mdx323

9. Yao X, Corbett T, Gupta AA, et al. A systematic review of active treatment options in patients with desmoid tumours. Curr Oncol. 2014;21(4):e613-629. doi:10.3747/co.21.1995

10. Gronchi A, Colombo C, Le Péchoux C, et al. Sporadic desmoid-type fibromatosis: a stepwise approach to a non-metastasising neoplasm a position paper from the Italian and the French sarcoma group. Ann Oncol. 2014;25(3):578-583. doi:10.1093/annonc/mdt485

11. Keus RB, Nout RA, Blay JY, et al. Results of a Phase II pilot study of moderate dose radiotherapy for inoperable desmoid-type fibromatosis - an EORTC STBSG and ROG study (EORTC 62991-22998). Ann Oncol. 2013;24(10):2672-2676. doi:10.1093/ annonc/mdt 254

12. Fiore M, Colombo C, Radaelli S, et al. Hormonal manipulation with toremifene in sporadic desmoid-type fibromatosis. Eur J Cancer. 2015;51(18):2800-2807. doi:10.1016/j.ejca.2015.08.026

13. Wang Z, Wu J, Tian X, Hao C. Targeted therapy of desmoid-type fibromatosis: mechanism, current situation, and future prospects. Front Med. 2019;13(4):427-437. doi:10.1007/s11684-018-0672-6

14. Elnekave E, Atar E, Amar S, et al. Doxorubicin-eluting intra-arterial therapy for pediatric extra-abdominal desmoid fibromatoses: a promising approach for a perplexing disease. J Vasc Interv Radiol. 2018;29(10):1376-1382. doi:10.1016/j.jvir.2018.04.009

15. Palassini E, Frezza AM, Mariani L, et al. Long-term efficacy of methotrexate plus vinblastine/vinorelbine in a large series of patients affected by desmoid-type fibromatosis. Cancer J. 2017;23(2):86-91. doi:10.1097/PPO.0000000000000254

16. Penel N, Coindre JM, Bonvalot S, et al. Management of desmoid tumours: a nationwide survey of labelled reference centre networks in France. Eur J Cancer. 2016;58:90-96. doi:10.1016/j.ejca.2016. 02.008

17. Kasper B, Baumgarten C, Bonvalot S, et al. Management of sporadic desmoid-type fibromatosis: a European consensus approach based on patients' and professionals' expertise - a sarcoma patients EuroNet and European organisation for research and treatment of cancer/soft tissue and bone sarcoma group initiative. Eur J Cancer. 2015;51 (2):127-136.

18. Chugh R, Wathen JK, Patel SR, et al. Efficacy of imatinib in aggressive fibromatosis: results of a phase II multicenter sarcoma alliance for research through collaboration (SARC) trial. Clin Cancer Res. 2010;16(19):4884-4891. doi:10.1158/1078-0432.CCR-10-1177

19. Szucs Z, Messiou C, Wong HH, et al. Pazopanib, a promising option for the treatment of aggressive fibromatosis. Anticancer Drugs. 2017;28(4):421-426. doi:10.1097/CAD.0000000000000474

20. Gounder MM, Lefkowitz RA, Keohan ML, et al. Activity of sorafenib against desmoid tumor/deep fibromatosis. Clin Cancer Res. 2011;17(12):4082-4090. doi:10.1158/1078-0432.CCR-10-3322
21. Gounder MM, Mahoney MR, Van Tine BA, et al. Sorafenib for advanced and refractory desmoid tumors. $N$ Engl J Med. 2018;379 (25):2417-2428. doi:10.1056/NEJMoa1805052

22. Sun Y, Niu W, Du F, et al. Safety, pharmacokinetics, and antitumor properties of anlotinib, an oral multi-target tyrosine kinase inhibitor, in patients with advanced refractory solid tumors. J Hematol Oncol. 2016;9(1):105. doi:10.1186/s13045-016-0332-8

23. Chi Y, Fang Z, Hong X, et al. Safety and efficacy of anlotinib, a multikinase angiogenesis inhibitor, in patients with refractory metastatic soft-tissue sarcoma. Clin Cancer Res. 2018;24(21):5233-5238. doi:10.1158/1078-0432.CCR-17-3766

24. Tang L, Yu W, Wang Y, Li H, Shen Z. Anlotinib inhibits synovial sarcoma by targeting GINS1: a novel downstream target oncogene in progression of synovial sarcoma. Clin Transl Oncol. 2019;21 (12):1624-1633. doi:10.1007/s12094-019-02090-2

25. Han B, Li K, Zhao Y, et al. Anlotinib as a third-line therapy in patients with refractory advanced non-small-cell lung cancer: a multicentre, randomised phase II trial (ALTER0302). $\mathrm{Br}$ $J$ Cancer. 2018;118(5):654-661. doi:10.1038/bjc.2017.478

26. Eisenhauer EA, Therasse P, Bogaerts J, et al. New response evaluation criteria in solid tumours: revised RECIST guideline (version 1.1). Eur J Cancer. 2009;45(2):228-247. doi:10.1016/j.ejca. 2008.10.026

27. Stacchiotti S, Collini P, Messina A, et al. High-grade soft-tissue sarcomas: tumor response assessment - pilot study to assess the correlation between radiologic and pathologic response by using RECIST and Choi criteria. Radiology. 2009;251(2):447-456. doi:10.1148/radiol.2512081403

28. Heinrich MC, McArthur GA, Demetri GD, et al. Clinical and molecular studies of the effect of imatinib on advanced aggressive fibromatosis (desmoid tumor). J Clin Oncol. 2006;24(7):1195-1203. doi:10.1200/JCO.2005.04.0717

29. Penel N, Le Cesne A, Bui BN, et al. Imatinib for progressive and recurrent aggressive fibromatosis (desmoid tumors): an FNCLCC/ French sarcoma group phase II trial with a long-term follow-up. Ann Oncol. 2011;22(2):452-457. doi:10.1093/annonc/mdq341

30. Kasper B, Gruenwald V, Reichardt P, et al. Imatinib induces sustained progression arrest in RECIST progressive desmoid tumours: final results of a phase II study of the German interdisciplinary sarcoma group (GISG). Eur J Cancer. 2017;76:60-67. doi:10.1016/j. ejca.2017.02.001

31. Wilhelm SM, Carter C, Tang L, et al. BAY 43-9006 exhibits broad spectrum oral antitumor activity and targets the RAF/MEK/ERK pathway and receptor tyrosine kinases involved in tumor progression and angiogenesis. Cancer Res. 2004;64(19):7099-7109. doi:10.1158/ 0008-5472.CAN-04-1443

32. Shen G, Zheng F, Ren D, et al. Anlotinib: a novel multi-targeting tyrosine kinase inhibitor in clinical development. J Hematol Oncol. 2018;11(1):120.

33. Wang HY, Chu JF, Zhang P, et al. Safety and efficacy of chemotherapy combined with anlotinib plus anlotinib maintenance in Chinese patients with advanced/metastatic soft tissue sarcoma. Onco Targets Ther. 2020;13:1561-1568. doi:10.2147/OTT.S235349

34. Luo J, Jin K, Qian S, et al. Single institution experience of split course radiotherapy in patients with desmoid tumors. Onco Targets Ther. 2019;12:1741-1748. doi:10.2147/OTT.S189449

35. Walker EA, Fenton ME, Salesky JS, Murphey MD. Magnetic resonance imaging of benign soft tissue neoplasms in adults. Radiol Clin North Am. 2011;49(6):1197-1217. doi:10.1016/j.rcl.2011. 07.007

36. Gondim Teixeira PA, Biouichi H, Abou Arab W, et al. Evidencebased MR imaging follow-up strategy for desmoid-type fibromatosis. Eur Radiol. 2020;30(2):895-902. doi:10.1007/s00330-019-06404-4

37. Sheth PJ, Del Moral S, Wilky BA, et al. Desmoid fibromatosis: MRI features of response to systemic therapy. Skeletal Radiol. 2016;45 (10):1365-1373. doi:10.1007/s00256-016-2439-y 


\section{Publish your work in this journal}

Drug Design, Development and Therapy is an international, peerreviewed open-access journal that spans the spectrum of drug design and development through to clinical applications. Clinical outcomes, patient safety, and programs for the development and effective, safe, and sustained use of medicines are a feature of the journal, which has also been accepted for indexing on PubMed Central. The manuscript management system is completely online and includes a very quick and fair peer-review system, which is all easy to use. Visit http://www. dovepress.com/testimonials.php to read real quotes from published authors. 